\title{
Interaction of the Macrolide Antibiotic Azithromycin with Lipid Bilayers: Effect on Membrane Organization, Fluidity, and Permeability
}

\author{
A. Berquand, ${ }^{1}$ N. Fa, ${ }^{2}$ Y. F. Dufrêne, ${ }^{1}$ and M.-P. Mingeot-Leclercq ${ }^{2,3}$
}

\begin{abstract}
Received July 9, 2004; accepted December 6, 2004
Purpose. To investigate the effect of a macrolide antibiotic, azithromycin, on the molecular organization of DPPC:DOPC, DPPE:DOPC, SM:DOPC, and SM:Chol:DOPC lipid vesicles as well as the effect of azithromycin on membrane fluidity and permeability.

Methods. The molecular organization of model membranes was characterized by atomic force microscopy (AFM), and the amount of azithromycin bound to lipid membranes was determined by equilibrium dialysis. The membrane fluidity and permeability were analyzed using fluorescence polarization studies and release of calcein-entrapped liposomes, respectively.

Results. In situ AFM images revealed that azithromycin leads to the erosion and disappearance of DPPC and DPPE gel domains, whereas no effect was noted on SM and SM:cholesterol domains. Although azithromycin did not alter the permeability of DPPC:DOPC, DPPE:DOPC, SM:DOPC, and SM:Chol:DOPC lipid vesicles, it increased the fluidity at the hydrophilic/hydrophobic interface in DPPC:DOPC and DPPE:DOPC models. This effect may be responsible for the ability of azithromycin to erode the DPPC and DPPE gel domains, as observed by AFM.

Conclusions. This study shows the interest of both AFM and biophysical methods to characterize the drug-membrane interactions.
\end{abstract}

KEY WORDS: AFM; azithromycin; fluidity; lateral domains; lipids; permeability.

\section{INTRODUCTION}

In the past two decades there has been considerable advance in our knowledge of membrane structure. The fluid mosaic model of Singer and Nicolson (1) has had great heuristic value in thinking about membrane topology (2). In this model, both proteins and lipids are free to diffuse in the bilayer, implying a random organization of protein and lipid. However, experimental evidence in diverse types of membranes and for several protein entities and lipids indicates that they may be organized in domains (3-6). The concept of the organization of the lipid components of membranes into domains raises, however, several questions. In a pharmaceutical context, one important question is to know if amphiphilic or lipophilic drugs preferentially partition and segregate into specific domains, and may such unique partitioning predicate specific functional effects?

Earlier, a number of drugs have been described to alter lipid order and domain formation. Examples include anesthetics $(7,8)$, cyclosporine (9), steroids (10), trifluoroperazine (11), and antibiotics like aminoglycosides (12) or macrolides (azithromycin) (13). This latter compound was also shown to

\footnotetext{
${ }^{1}$ Unité de Chimie des Interfaces, Université Catholique de Louvain, B-1348 Louvain-la-Neuve, Belgium.

${ }^{2}$ Unité de Pharmacologie Cellulaire et Moléculaire, Université Catholique de Louvain, B-1200 Brussels, Belgium.

${ }^{3}$ To whom correspondence should be addressed. (e-mail: mingeot@ facm.ucl.ac.be)
}

markedly inhibit endocytosis $(14,15)$, probably by interacting with lipids, modifying biophysical properties of membrane and affecting membrane dynamics in living cells (13).

In recent years, atomic force microscopy (AFM) has been used for characterizing the structural properties of supported lipid films in terms of molecular structure, lipid domain formation, and interaction with external pharmaceutical agents (16-18). AFM is a powerful tool for probing biological specimens at high resolution (19). Because the technique can be operated in aqueous solutions, the samples can be examined directly under physiologic conditions.

In this study, using AFM imaging, we investigated the effect of azithromycin on the molecular organization of model membranes of DPPC:DOPC, DPPE:DOPC, SM:DOPC, and SM:Chol:DOPC. In parallel, we gained data by equilibrium dialysis and fluorescence polarization studies on the amount of the drug bound to lipid membranes and its effect on membrane fluidity. Because difference in lateral membrane organization can lead to instabilities and structure defects in the boundary regions, which would be expected to cause increased permeability, we also investigated the effect of azithromycin on the release of calcein entrapped in liposomes as a reflect of membrane permeability. We showed that the influence of the drug on lipidic domains strongly depends on the lipid nature. Azithromycin is able to erode gel domains of DPPC and DPPE domains embedded in a fluid DOPC matrix. By contrast, SM-containing domains are not eroded in the same conditions. Absence of effect on membrane fluidity at the hydrophobic/hydrophilic interface and/or partial exclu- 
sion of the drug could explain the inability of azithromycin to erode the SM-containing domains.

\section{MATERIALS AND METHODS}

Azithromycin was supplied as dihydrate free base $\left(\mathrm{M}_{\mathrm{w}}\right.$ 785) by Pfizer (Groton, CT, USA). Azithromycin is sparingly soluble in water at $\mathrm{pH}=7.4$. A stock solution was prepared by dissolving $22.5 \mathrm{mg}$ of the free base in $1 \mathrm{ml} 0.1 \mathrm{M} \mathrm{HCl}(28.6$ $\mathrm{mM}$ ) and was diluted in Tris: $\mathrm{NaCl} 10: 100 \mathrm{mM} \mathrm{pH} 7.4$ buffer to a final concentration of $0.79 \mathrm{mg} / \mathrm{ml}(1 \mathrm{mM})$. Glycerophospholipids (dioleoylphosphatidylcholine [DOPC], dipalmitoyl phosphatidylcholine [DPPC], dipalmitoylphosphatidyletanolamine [DPPE]), sphingolipid (sphingomyelin from bovine brain $[\mathrm{SM}]$ ), cholesterol (Chol), calcein, and mellitin were purchased from Sigma (St. Louis, MO, USA). Tris (Tris-hydroxymethyl-aminomethan), $\mathrm{NaCl}$ (sodium chloride), and $\mathrm{CaCl}_{2} \cdot 2 \mathrm{H}_{2} \mathrm{O}$ (calcium chloride dihydrate) were purchased from Merck (Darmstadt, Germany). Diphenylhexatriene (DPH) and trimethylammoniumdiphenylhexatriene (TMADPH) were obtained from Molecular Probes (Eugene, OR, USA).

\section{Preparation of Liposomes (SUVs)}

Small unilamellar vesicles (SUVs) consisting of DOPC, DPPC:DOPC (1:1; mol:mol), DPPE:DOPC (1:1; mol:mol), SM:DOPC (1:1; mol:mol), or SM:Chol:DOPC (1:1:1; mol: mol:mol) were prepared as follows. Lipids were mixed in $\mathrm{CHCl}_{3}: \mathrm{MeOH}$ 2:1 (v:v), evaporated under nitrogen flow, and dessicated under vacuum for $2 \mathrm{~h}$. The dried films were then resuspended at room temperature from the walls of the glass tube by vigorous vortexing either in Tris: $\mathrm{NaCl}$ buffer 10:100 $\mathrm{mM}, \mathrm{pH} 7.4$ for quasi-elastic light scattering, equilibrium dialysis, fluorescence polarization, and permeability studies or in Tris: $\mathrm{NaCl}: \mathrm{CaCl}_{2} \cdot 2 \mathrm{H}_{2} \mathrm{O}$ buffer 10:100:3 mM, pH 7.4 for AFM studies. The final concentration of lipids was $10 \mathrm{mg} / \mathrm{ml}$ for AFM and equilibrium dialysis, $2 \mathrm{mg} / \mathrm{ml}$ for permeability studies, $0.5 \mathrm{mg}$ for quasi-elastic light scattering analysis, and $0.2 \mathrm{mg} / \mathrm{ml}$ for fluorescence polarization studies. The preparation, cooled by an ice bath, was sonicated to clarity 5 times for 2 min each using a Fisher Bioblock Scientific $750 \mathrm{~W}$ sonicator (Avantec, Illkirch, France) set at $35 \%$ of the maximal power, and a $13 \mathrm{~mm}$ probe. The SUVs preparation was filtered on $0.2-\mu \mathrm{m}$ Acrodisc filters to eliminate titanium particles.

\section{Quasi-elastic Light Scattering Studies}

The apparent average diameter of small unilamellar vesicles was determined at room temperature by quasi-elastic light scattering spectroscopy using a Coulter Nano Sizer $\mathrm{N}_{4} \mathrm{MD}$ (Coulter Electronics Ltd, Luton, UK), as described earlier (20). The liposomes concentration was set at $0.5 \mathrm{mg} / \mathrm{ml}$.

Fluctuation of light scattering was measured at an angle of 90 degrees with monodisperse latex particles of 100 and $800 \mathrm{~nm}$ diameter as control. Data were analyzed using size distribution analysis mode to determine the full size distribution profile of liposomes mixed with azithromycin.

\section{Atomic Force Microscopy}

Mica sheets were heated $1 \mathrm{~h}$ before the fusion at $60^{\circ} \mathrm{C}$ and cleaved to obtain a flat and uniform surface. The SUV suspension, was put into contact with the mica surface for $45 \mathrm{~min}$ at $60^{\circ} \mathrm{C}$ and the sample was slowly cooled back to ambiant temperature to prevent thermal shock. The excess of SUVs was then eliminated by 4 times rinsing with a Tris: $\mathrm{NaCl}$ 10:100 $\mathrm{mM}$ buffer or solution containing $1 \mathrm{mM}$ azithromycin. The sample was installed on the microscope without dewetting. The liquid meniscus was completed with the same buffer. All AFM measurements were carried out at room temperature in contact mode using an optical detection system equipped with a liquid cell (Nanoscope III; Digital Instruments, Santa Barbara, CA, USA). Topographic images were taken in the constant-deflection mode using oxide-sharpened microfabricated $\mathrm{Si}_{3} \mathrm{~N}_{4}$ cantilevers (Park Scientific Instruments, Mountain View, CA, USA) with typical curvature radii of $20 \mathrm{~nm}$ and spring constant of 0.01 and $0.03 \mathrm{~N} / \mathrm{m}$. Scan rate ranging from 3 to $5 \mathrm{~Hz}$ were tested. The applied force was maintained as low as possible $(<1 \mathrm{nN})$ during the imaging.

\section{Equilibrium Dialysis Studies}

Binding of azithromycin to SUVs prepared in Tris: $\mathrm{NaCl}$ 10:100 mM pH 7.4 was investigated by equilibrium dialysis using a Dianorm apparatus (Dianorm Geraäte, Munchen, Germany), consisting of sets of $200 \mu l$ twin cells made of Teflon and separated by a Diachema flat dialysis membrane (Mr cutoff: 5000) as described earlier (20). The drug was introduced in one chamber at a $120 \mu \mathrm{M}$ concentration $\left(\mathrm{D}_{\text {initial }}\right)$, and SUVs in the other chamber at a total lipid concentration of $10 \mathrm{mg} / \mathrm{ml}$. Dialysis was performed overnight at room temperature under constant rotation (5 rpm) (control experiments showed that equilibrium dialysis was achieved after $3 \mathrm{~h}$ ). In the chamber containing no SUVs, azithromycin was assayed after dialysis $\left(D_{\text {final }}=D_{\text {free }}\right)$ by a disk-plate microbiological assay using Bacillus subtilis. The concentration of the drug in the chamber containing liposomes $\left(D_{\text {free }}+D_{\text {bound }}\right.$ $\left.\left[\left(D_{\text {total }}\right]\right)\right)$ was calculated as $D_{\text {initial }}-D_{\text {free }}$.

\section{Fluorescence Polarization Studies}

Fluorescence polarization studies were performed on SUVs prepared in Tris: $\mathrm{NaCl}$ 10:100 mM pH 7.4 buffer, at a final concentration of $0.2 \mathrm{mg} / \mathrm{ml}$. Incorporation of fluorescent markers (at a molar ratio to the lipids of 1:250) was obtained by a vigorous mixing followed by a preincubation at $37^{\circ} \mathrm{C}$ during $1 \mathrm{~h}$. Two markers with distinct localization in the lipid bilayer were used for comparison (diphenylhexatriene [DPH] a totally hydrophobic probe, which penetrates deeply into the membrane, and trimethylammoniumdiphenylhexatriene [TMA-DPH], which spans the hydrophilic/hydrophobic interface because of its amphiphilic character (21-23).

SUVs were mixed with azithromycin, incubated at $37^{\circ} \mathrm{C}$ for $1 \mathrm{~h}$, brought to $60^{\circ} \mathrm{C}$ in $15 \mathrm{~min}$, and maintained at that temperature during $5 \mathrm{~min}$ for stabilization before starting the measurements. The fluorescence emitted in the planes parallel $\left(\mathrm{I}_{\mathrm{par}}\right)$ and perpendicular $\left(\mathrm{I}_{\mathrm{per}}\right)$ to that of the polarized excitation light was then measured while the samples were cooled to $10^{\circ} \mathrm{C}$ at a rate of $50^{\circ} \mathrm{C} / \mathrm{h}$. Results are expressed as $\mathrm{P}=\left(\mathrm{I}_{\mathrm{par}}-\mathrm{I}_{\mathrm{per}}\right) /\left(\mathrm{I}_{\mathrm{par}}+\mathrm{I}_{\mathrm{per}}\right)$. Fluorescence was measured on a LS-50 Perkin-Elmer fluorimeter (Perkin-Elmer, Beaconsfield, UK), equipped with a special adaptator for polarization measurements, and operating at an excitation wavelength of $365 \pm 5 \mathrm{~nm}$ (for DPH) or $360 \pm 5 \mathrm{~nm}$ (for TMA-DPH) and an 
emission wavelength of $427 \pm 3 \mathrm{~nm}$ (for DPH) or $435 \pm 4 \mathrm{~nm}$ (for TMA-DPH). The sample was kept under gentle stirring throughout the experiment, and its temperature was continuously monitored by a sensor placed into the measuring unit coupled with a programmable circulator bath DC5 (Haake, Karlsruhe, Germany).

\section{Release of Calcein-Entrapped in Liposomes}

Leakage of entrapped, self-quenched-calcein from SUVs was monitored by the increase of fluorescence subsequent to dilution (24). The dry lipid films were hydrated to a final lipid concentration of $2 \mathrm{mg} / \mathrm{ml}$ in a solution of purified calcein. The final solution had an osmolarity of $353 \mathrm{mosm} / \mathrm{kg}$ (measured by the freezing point technique [Advanced Cryomatic osmometer, model 3C2, Advanced Instruments Inc., Needham Heights, MA, USA]). After the preparation of vesicles, the unencapsulated dye was discarded using the minicolumn centrifugation technique of Lelkes as described previously (25). The recovery of liposomes was determined by measuring their phospholipid content, using phosphorus assay (26) and was typically $>90 \%$. The liposomes were diluted to a final lipid concentration of $50 \mu \mathrm{M}$ in isoosmotic Tris buffer $\mathrm{pH} 7.4$ (353 mOsm $/ \mathrm{kg})$. Azithromycin $(7 \mu \mathrm{M})$, or melittin, used as positive control, were added to the liposomes. The mixture was vortexed for $20 \mathrm{~s}$ and the first fluorescence determination was made $1 \mathrm{~min}$ after addition of the drug. All fluorescence determinations were performed at room temperature on a Perkin Elmer LS 30 Fluorescence Spectrophotometer (Perkin-Elmer Ltd.) using excitation and emission wavelengths of $472 \mathrm{~nm}$ and $516 \mathrm{~nm}$, respectively. The percentage of calcein released under the influence of azithromycin (or mellitin) was defined as $\left[\left(F_{t}-F_{\text {contr }}\right) /\left(F_{\text {tot }}-F_{\text {contr }}\right)\right] \times 100$, where $F_{t}$ is the fluorescence signal measured at time $(\mathrm{t})$ in the presence of the drug, $F_{\text {contr }}$ is the fluorescence signal measured at the same time in the control liposomes, and $F_{\text {tot }}$ is the total fluorescence signal obtained after complete disruption of liposomes by ultrasound (verified by quasi-elastic light scattering) which caused complete release of calcein.

\section{RESULTS}

\section{Size of the Liposomes}

Previous to the study of the effect of azithromycin on the membrane organization, fluidity, and permeability, we investigated by quasi-elastic light scattering the size and the homogeneity of the different liposomes used (DOPC, DPPC: DOPC, DPPE:DOPC, SM:DOPC, and SM:Chol:DOPC) (Table I). The results showed no major effect of the composition on the apparent diameter and homogeneity of the preparation. The obtained $\sim 80-90 \mathrm{~nm}$ size is somewhat larger than what is typically expected for SUV liposomes, but this may be accounted by the fact that this type of measurement is strongly influenced by the presence of a small number of large particles.

\section{Effect of Azithromycin on Membrane Organization}

AFM was used to investigate the morphology of mixed DPPC:DOPC, DPPE:DOPC, SM:DOPC, and SM:Chol:DOPC bilayers in the absence and presence of azithromycin. Figure 1 shows that, in the absence of azithromycin, all bilayers displayed two discrete height levels in the topographic images reflecting phase separation between solid-like DPPC, DPPE, SM, and SM:Chol domains and liquid-like DOPC. The DPPC gel domains were well defined and homogenous, with a size ranging from $150 \mathrm{~nm}$ to $1.5 \mu \mathrm{m}$ (Fig. 1A). The height difference between DPPC domains and the fluid DOPC matrix was $1.10 \pm 0.05 \mathrm{~nm}$. As opposed to DPPC, DPPE gel domains appeared as interconnected features with variable morphologies (Fig. 1B), the height difference between gel and fluid phase ranging from 0.80 to $1.20 \mathrm{~nm}$. The SM:DOPC system showed the smallest domains, their average size being in the 50-500 $\mathrm{nm}$ range (Fig. 1C). The height difference between gel and fluid phases was $0.85 \pm 0.03 \mathrm{~nm}$. Finally, when cholesterol was added to SM:DOPC bilayers, a 10-fold increase in the cholesterol-rich domain size was noted, compared to SM: DOPC bilayers, while the height difference between the two phases was similar $(0.85 \pm 0.05 \mathrm{~nm})$ (Fig. 1D).

Scanning lipid films with the AFM tip may alter the image contrast or create artificial features that were not initially present in the bilayer $(27,28)$. To assess whether such alterations may occur in our systems, the same areas were scanned during $1 \mathrm{~h}$ while minimizing continuously the applied force. After 60 min scanning, the morphology of the DPPC, SM, or SM:Chol domains remained unchanged while the DPPE domains disappeared from the scanned area, suggesting they were much more fragile than the other domains (data not shown).

New supported bilayers, prepared from the different lipid mixtures, were then directly incubated with a $1 \mathrm{mM}$ azithromycin solution. At short contact time (Figs. $2 \mathrm{~A}$ and C, 3 A and C), the morphology of DPPC:DOPC, DPPE:DOPC, SM:DOPC, and SM:Chol:DOPC bilayers was not or slightly modified compared to the native bilayers.

In contrast, after $60 \mathrm{~min}$, the morphology was strongly affected, and this effect was influenced by the nature of the lipid. A complete disappearance of DPPC and DPPE domains from the scanned area (Fig. 2 B and D) was observed, whereas SM and SM:Chol domains remained totally unchanged in the presence of azithromycin (Fig. $3 \mathrm{~B}$ and D).

Table I. Size of Liposomes as Determined by Quasi-elastic Light Scattering

\begin{tabular}{lccccc}
\hline & \multicolumn{5}{c}{ Composition } \\
\cline { 2 - 6 } & DOPC & $\begin{array}{c}\text { DOPC:DPPC } \\
(1: 1 \mathrm{~mol}: \mathrm{mol})\end{array}$ & $\begin{array}{c}\text { DOPC:DPPE } \\
(1: 1 \mathrm{~mol}: \mathrm{mol})\end{array}$ & $\begin{array}{c}\text { DOPC:SM } \\
(1: 1 \mathrm{~mol}: \mathrm{mol})\end{array}$ & $\begin{array}{c}\text { DOPC:Chol:SM } \\
(1: 11 \mathrm{~mol}: \mathrm{mol}: \mathrm{mol})\end{array}$ \\
\hline Diameter & $85 \mathrm{~nm}(95 \%)$ & $70 \mathrm{~nm}(94 \%)$ & $89 \mathrm{~nm} \mathrm{(92 \% )}$ & $87 \mathrm{~nm}(93 \%)$ & $88 \mathrm{~nm}(91 \%)$ \\
(Percentage) & $341 \mathrm{~nm}(5 \%)$ & $384 \mathrm{~nm}(6 \%)$ & $387 \mathrm{~nm} \mathrm{(8 \% )}$ & $350 \mathrm{~nm} \mathrm{(7 \% )}$ & $703 \mathrm{~nm} \mathrm{(9 \% )}$ \\
\hline
\end{tabular}



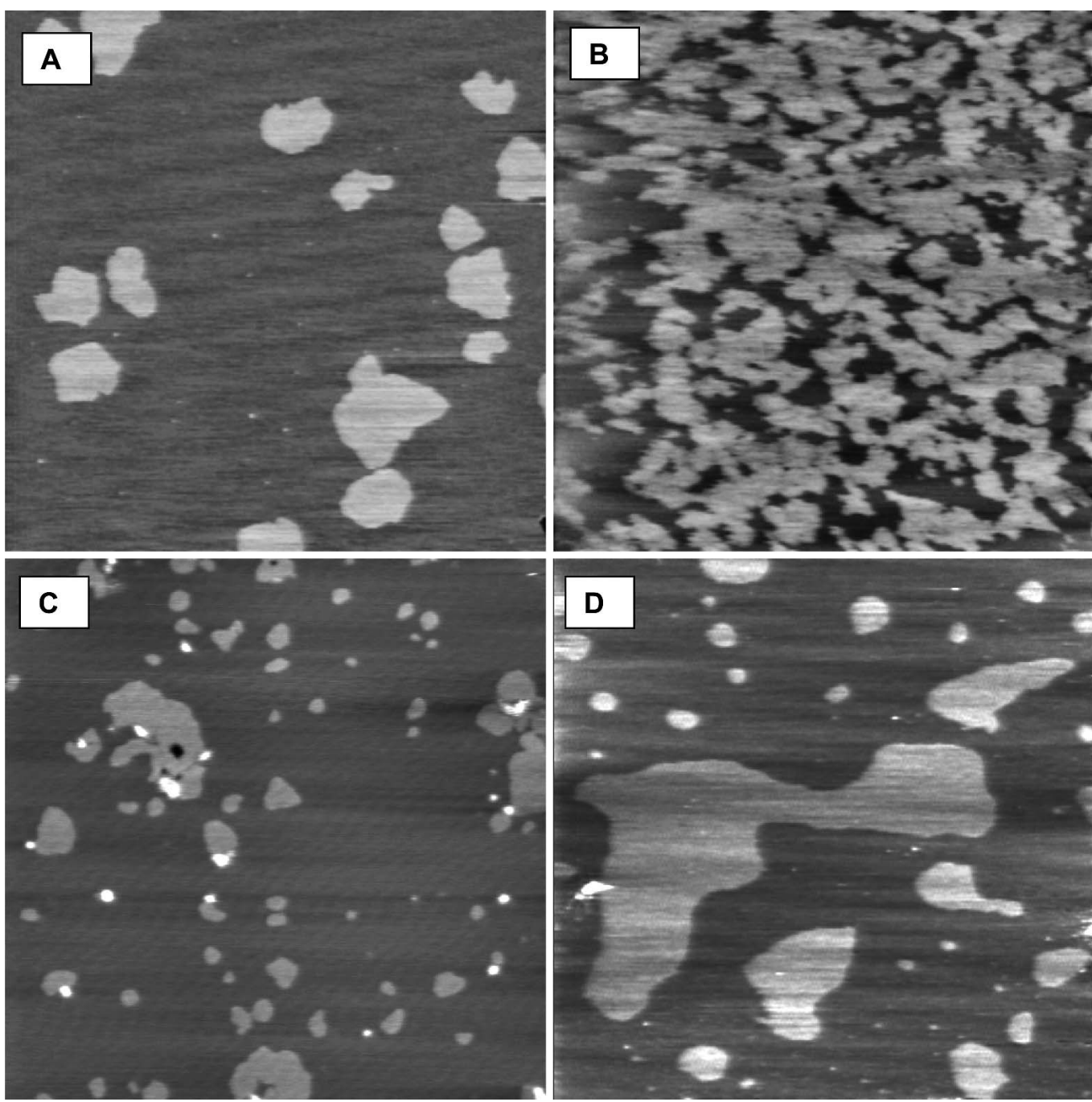

Fig. 1. AFM height images $(5 \mu \mathrm{m} \times 5 \mu \mathrm{m}$; z-scale: $10 \mathrm{~nm})$ of mixed lipid bilayers recorded in buffer solution: (A) DPPC:DOPC (1:1, mol:mol), (B) DPPE:DOPC (1:1, mol:mol), (C) SM:DOPC (1:1, mol:mol), and (D) SM:Chol:DOPC (1:1:1, mol:mol:mol). Data are representative of four experiments.

\section{Binding of Azithromycin to Liposomes}

Binding of azithromycin to small unilamellar vesicles made of the different lipid mixtures investigated was measured by equilibrium dialysis at pH 7.4 (Fig. 4).

At an initial drug concentration of $120 \mu \mathrm{M}, 9.12 \% \pm 1.00$ of azithromycin bound to SUVs composed of pure DOPC. In the case of a DPPC:DOPC mixture, the bound fraction increased up to $11.64 \% \pm 0.77$. Replacing DPPC by DPPE or SM slightly decreased the bound fraction to $9.87 \% \pm 1.12$ and $8.86 \% \pm 0.75$, respectively. Finally using a ternary mixture constituted of SM:Chol:DOPC 1:1:1, only $6.24 \% \pm 1.60$ of azithromycin bound to SUVs. This binding corresponded to a molecular ratio between lipid and azithromycin of 1162:1 (DOPC), 942:1 (DPPC:DOPC), 1142:1 (DPPE:DOPC), 1240: 1 (SM:DOPC), and 635:1 (SM:Chol:DOPC).

\section{Effect of Azithromycin on Membrane Fluidity}

To investigate the influence of azithromycin on membrane fluidity, we examined its effect on the fluorescence polarization of 1,6-diphenylhexatriene (DPH) (Fig. 5, left panels) and of its protonated derivative, trimethylammonium diphenylhexatriene (TMA-DPH) (Fig. 5, right panels). Results showed that the degree of polarization of the two probes decreased linearly in control liposomes when the temperature of the sample was increased (29). Except for vesicles containing cholesterol, the polarization value recorded with TMADPH was systematically higher than with DPH, confirming the intrinsic higher rigidity of the interface as compared to the hydrophobic region of the membrane.

The addition of azithromycin did not change significantly the degree of polarization of DPH (Fig. 5 A-D) inserted in cholesterol-free vesicles. For ternary mixtures of SM:Chol:DOPC 1:1:1 (Fig. 5E), addition of azithromycin reduced the polarization values, especially at low temperature. On the contrary, considering the variation of the polarization of the TMA-DPH, azithromycin seemed to have no influence on the ternary system (Fig. 5J), whereas it increased the fluidity of the hydrophobic/hydrophilic interface of DOPC, DPPC:DOPC, and DPPE:DOPC mixture (Fig. $5 \mathrm{~F}-\mathrm{H}$ ) and slightly induced rigidity of SM:DOPC (Fig. 5I). The effect observed on DPPC:DOPC seemed dependent of the temperature (Fig. 5G). 

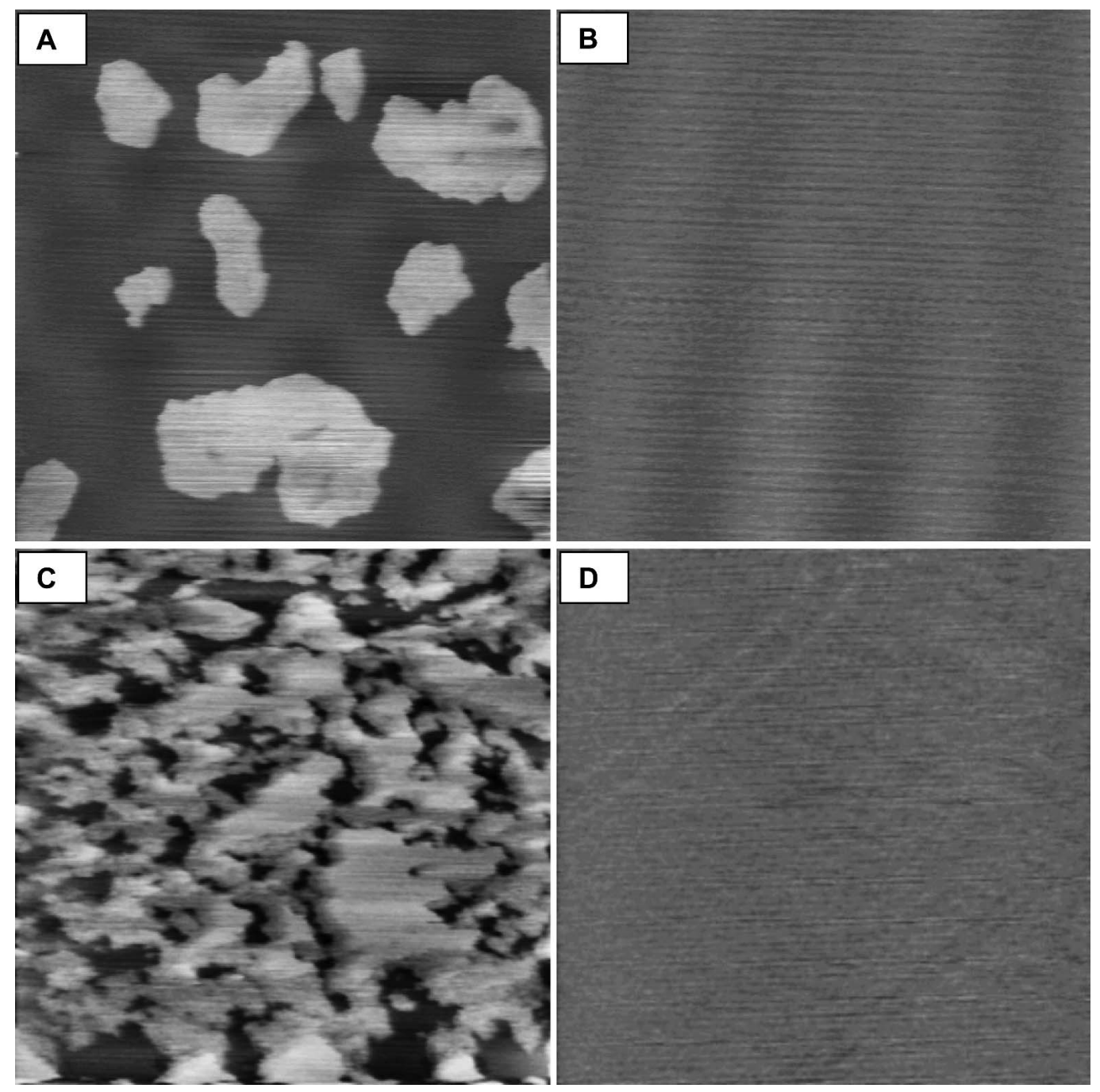

Fig. 2. AFM height images $(5 \mu \mathrm{m} \times 5 \mu \mathrm{m}$; z-scale: $10 \mathrm{~nm})$ recorded for mixed DPPC:DOPC (1:1, mo:/mol) $(A$, B) and DPPE:DOPC (1:1, mol:mol) (C, D) bilayers after 1 min (A, C) and $60 \mathrm{~min}(\mathrm{~B}, \mathrm{D})$ contact time with azithromycin $(1 \mathrm{mM})$. Data are representative of three experiments.

\section{Effect of Azithromycin on Membrane Permeability}

SUVs containing calcein entrapped at self-quenching concentrations, were incubated with azithromycin at room temperature or at $45^{\circ} \mathrm{C}$. Calcein is a polar molecule that has been widely used to study the permeability of lipid bilayers and has been originally described for this application by Weinstein (24).

The effect induced by azithromycin is shown on Fig. 6. In contrast to mellitin, which induced $95 \%$ of leakage of entrapped calcein in the first minutes of incubation, whatever the lipidic composition of the vesicules investigated, azithromycin did not enhance the release of calcein-entrapped in DPPC:DOPC, DPPC:DPPE, SM:DOPC, and SM:Chol:DOPC vesicles. For DOPC vesicles, only a slow release was observed (around $30 \%$ ). No difference was found in function of the temperature.

\section{DISCUSSION}

One of the major goals of current research in biophysics is to characterize the fundamental interactions between drugs or macromolecules and multimolecular structures such as lipid monolayers or bilayers (30-36). Formation of lateral microdomains is particularly important in this respect both from the structural and functional points of view. Generally speaking, lateral domains result from the presence of specific lipids like cholesterol (37), ceramide (38), lysobisphosphatidic acid (39), or proteins like phosphatidylinositol transfer proteins (40) or bacteriorhodopsin (41). The presence of defects or domains in the bilayer structure may act as starting points for the enzyme activity (42-45), entry of bacteria (46), budding and fission $(47,48)$, or apoptosis $(49)$. Microdomains have also been suggested to affect membrane permeability and from a pharmacological point of view play a key role in the phenomenon of drug-enhanced adsorption (50-52).

Using AFM, we showed that lipid mixtures of DPPC: DOPC, DPPE:DOPC, SM:DOPC, and SM:Chol:DOPC exhibit well defined phase separation. The shape and size of the domains varied from small disks $(150 \mathrm{~nm})$ to large $(3 \mu \mathrm{m})$, elongated structures. The height difference between gel domains and fluid matrix varied from $0.8 \mathrm{~nm}$ to $1.2 \mathrm{~nm}$, which is in good agreement with previous studies (53). This variation 

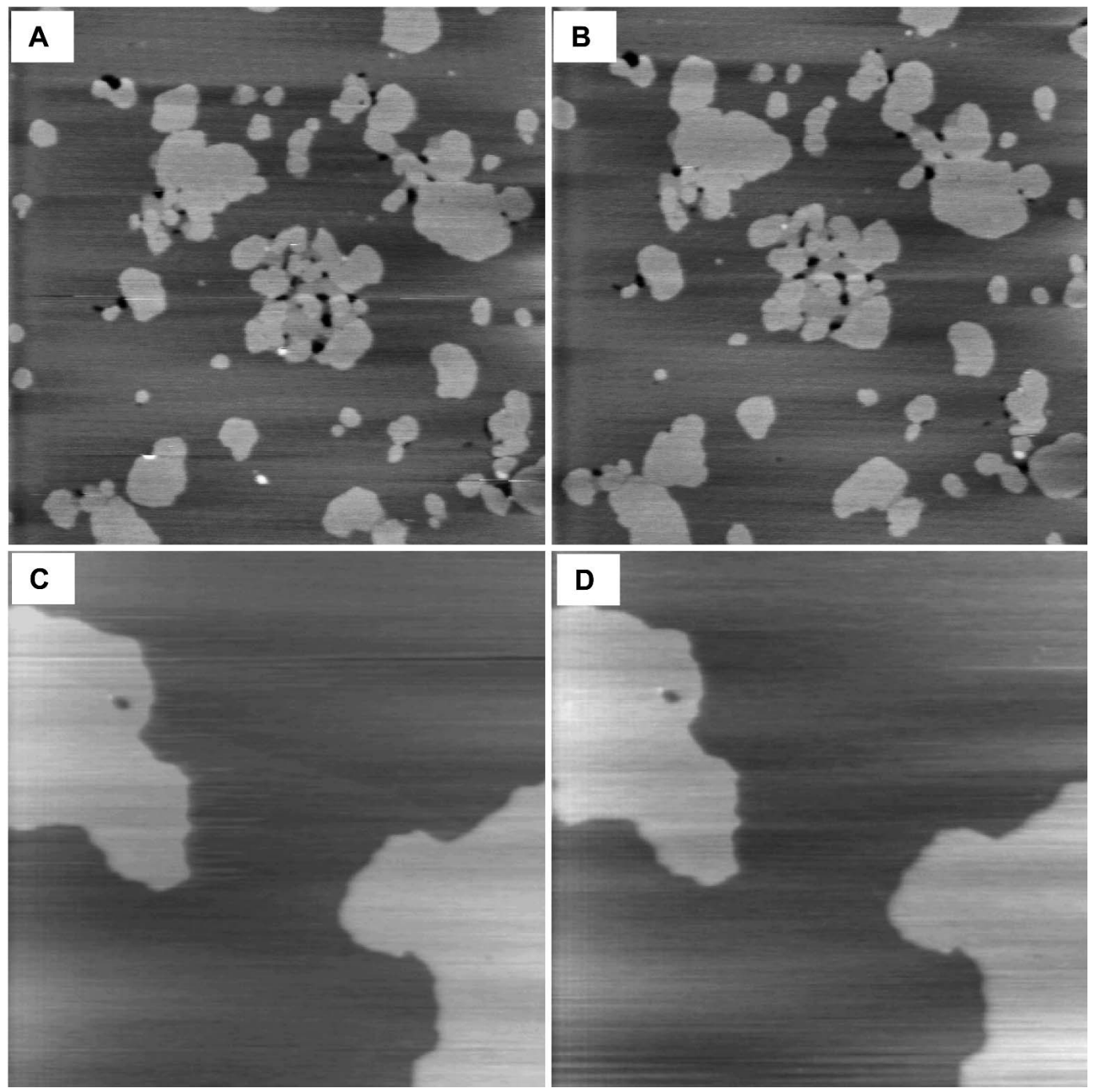

Fig. 3. AFM height images $(5 \mu \mathrm{m} \times 5 \mu \mathrm{m}$; z-scale: $10 \mathrm{~nm})$ recorded for mixed SM:DOPC $(1: 1$, mol:mol $)(A, B)$ and SM:Chol:DOPC (1:1:1, mol:mol:mol) (C, D) bilayers after $1 \mathrm{~min}(\mathrm{~A}, \mathrm{C})$ and $60 \mathrm{~min}(\mathrm{~B}, \mathrm{D})$ contact time with azithromycin $(1 \mathrm{mM})$. Data are representative of three experiments.

in domain shape and size illustrates the complexity of the phase separation process when observed at the mesoscopic scale (53).

The effect of azithromycin on the lipid domains was found to strongly depend on the lipid nature. Incubation of DPPC:DOPC bilayers with azithromycin for 60 min resulted in the complete disappearance of the gel domains. This erosion of the gel domains could be due to an interaction between azithromycin and DPPC molecules. Intercalation of the drug in the lipid layer would disrupt the interactions between the lipid polar headgroups, and possibly between the interfacial domains, thereby leading to a fluidification, and thus disappearance, of the domains.

These observations may be related to previous AFM work performed on phase-separated DPPC/cholesterol monolayers in air (13). Addition of azithromycin to the lipid mixture dramatically altered the film properties. Aggregates were observed at the mica surface, rather than a continuous film, and destabilization of the lipid system occurred as revealed by material reorganization upon scanning. It was suggested that the drug interacts with the DPPC molecules at the air/water interface, thereby altering the interaction of the monolayer with mica during transfer.

Disruption of bilayer domains was also observed for DPPE, but in this case the observed changes may be attributed to the combined effect of the drug and of the AFM tip. The higher fragility of DPPE domains in lipid bilayers is consistent with their tendency to form inverted micelles (54).

Our AFM results can be related to our data concerning the interaction between azithromycin and interfacial and hydrophobic part of the phospholipids, as examined by fluorescence polarization of trimethylammoniumdiphenylhexatriene (TMA-DPH) and diphenylhexatriene (DPH), respectively.

For DPPC:DOPC and DPPE:DOPC SUVs, azithromycin increased fluidity in the zone where TMA-DPH was localized. Comparing the behavior of DPPC and DPPE domains with that of SM domains, AFM images revealed that SM gel domains were not modified by azithromycin, even after $1 \mathrm{~h}$ scanning and fluorescence polarization studies of TMA-DPH showed no or only a very slight decrease of fluidity for SM:DOPC SUVs in the presence of azithromycin. Because no change of fluidity was observed in domains where 


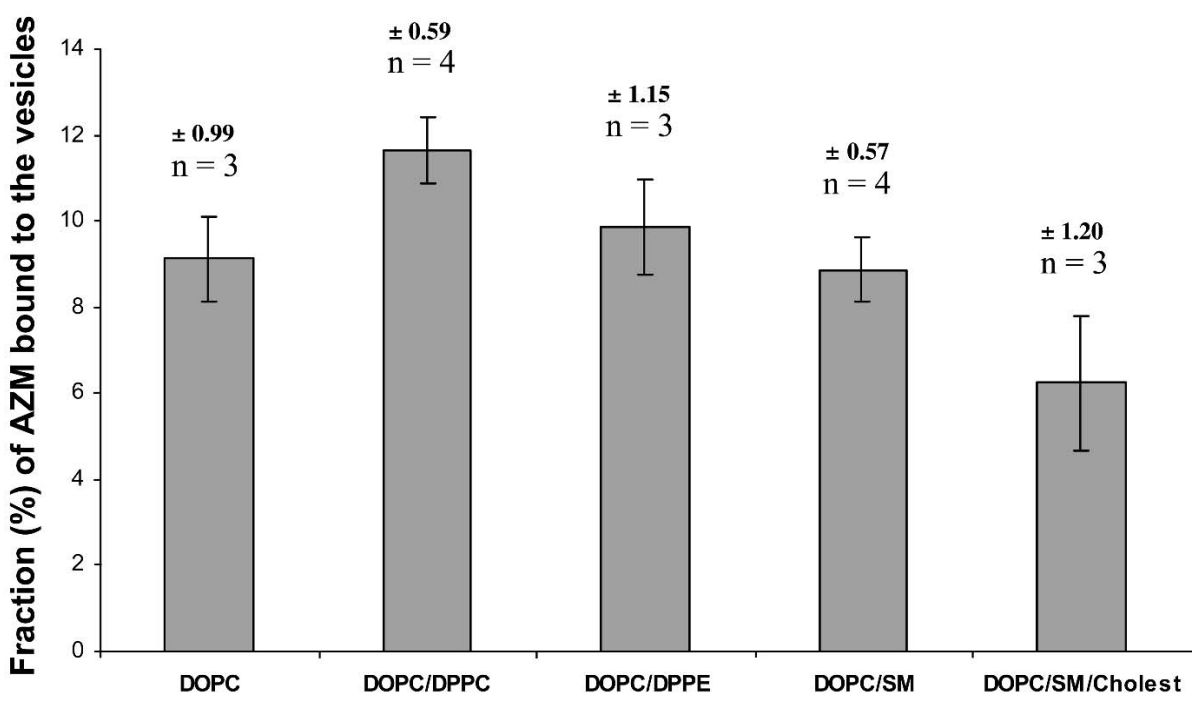

Fig. 4. Equilibrium dialysis of azithromycin $(120 \mu \mathrm{M})$, against lipid vesicles $(10 \mathrm{mg} / \mathrm{ml})$ of DOPC, DPPC:DOPC (1:1, mol:mol), DPPE:DOPC (1:1, mol:mol), SM:DOPC (1:1, mol:mol), and SM:Chol:DOPC (1:1:1, mol:mol:mol). Results are expressed as percentage of azithromycin bound to lipids and are mean of at least three independent experiments. Statistical analysis revealed a $p$ $<0.05$ for the binding of azithromycin to vesicles containing SM:Chol:DOPC as compared to vesicles of DOPC, DPPE:DOPC, and SM:DOPC. The $p$ value was $<0.005$ for the binding of azithromycin to vesicles containing SM:Chol:DOPC as compared to vesicles of DPPC:DOPC. Mean \pm SD.

DPH was located, and because DPH is generally accepted to partition equally between ordered and disordered regions of a membrane (55-57), we suggested that the primary interaction of azithromycin with the interfacial domain of phospholipids was the most crucial event to explain the disparition of domains induced by azithromycin.

Ternary mixtures like SM:Chol:DOPC can exhibit much more complex behavior (58) but more relevant to physiologic situations (59). Domains rich in glycosphingolipids and cholesterol are found in living cells acting like platforms of signaling pathways $(60,61)$. Because cholesterol preferentially interacts with lipids with long saturated acyl-chains, like naturally occurring sphingomyelins $(62,63)$, the higher domains consist of SM and cholesterol in the liquid ordered phase surrounded by lipids in the fluid phase. In the presence of cholesterol, we observed domains that are larger in size-as reported by (64) - and less irregular in shape than the domains formed in the absence of this sterol. Addition of $33 \%$ cholesterol to the bilayers also reduced the thickness from 0.7 to $0.4 \mathrm{~nm}$ as reported by others (64-66). Such a reduction likely involved both the decrease in bilayer thickness at the level of sphingomyelin-enriched domains (65) and the increase in the bilayer thickness of the DOPC enriched domains (67) promoted by cholesterol.

The Lo (liquid-ordered) phase arising from the interaction of cholesterol with highly saturated phospholipids or with sphingomyelin is characterized by lipids well packed in an ordered state (as in solid-gel phase) and a lateral diffusion almost as fast as in the fluid liquid-crystalline phase. This results in high values of fluorescence polarization that we found. This also agrees with measurements of the compression of bilayers showing that it is harder to compress cholesterol-rich membranes $(68,69)$. The larger area compressibility modulus of SM:Chol compared with DOPC for example would mean that more energy would be needed to separate the acyl chains and therefore would make it energetically unfavorable for azithromycin to partition into the SM:Chol domains. This could explain why, by equilibrium dialysis, only about half of azithromycin partitioned into SM:Chol:DOPC bilayers as compared to results obtained for DOPC, DPPC: DOPC, DPPE:DOPC, or SM:DOPC SUVs. At this stage, we have no clear explanation for the increase of fluidity induced by azithromycin in hydrophobic domains of SM:Chol:DOPC.

Our results extent previous observations performed with negatively charged liposomes at pH 5.4 (70-72) in which we reported by equilibrium dialysis that azithromycin binds to phosphatidylinositol-containing liposomes in a fashion dependent of the phosphatidylinositol content and of ionic strength, by ${ }^{31} \mathrm{P}$ NMR studies that azithromycin interacts with the phosphate heads of phospholipids, and by fluorescence polarization studies that it effectively penetrates across the hydrophobic-hydrophilic interface. Comparing, by simulation studies, the interaction with phosphatidylinositol monolayer of four other macrolides (erythromycin, roxithromycin, dirithromycin, and erythromycylamine) to that of azithromycin (71), we found that the position of the macrocycle is very similar, probably due to the fact that the cycle has no frank hydrophilic and hydrophobic regions like a phospholipid (73) or a cationic amphiphile like bis-( $\beta$-diethylaminoethyl ether) hexestrol (74). This suggested that all the data obtained in the current study could be extended to other macrolide antibiotics.

The alteration of lipid order and domain formation due to the presence of azithromycin and drugs in general in the bilayer could have a number of dramatic consequences for the macroscopic properties of the bilayer. It can be expected that the presence of lateral separations, would be associated with high membrane permeability. Especially, the increased amount of interface as observed in DPPE:DOPC mixture would make the bilayer much more leaky due to instability of 
DPH

TMA-DPH

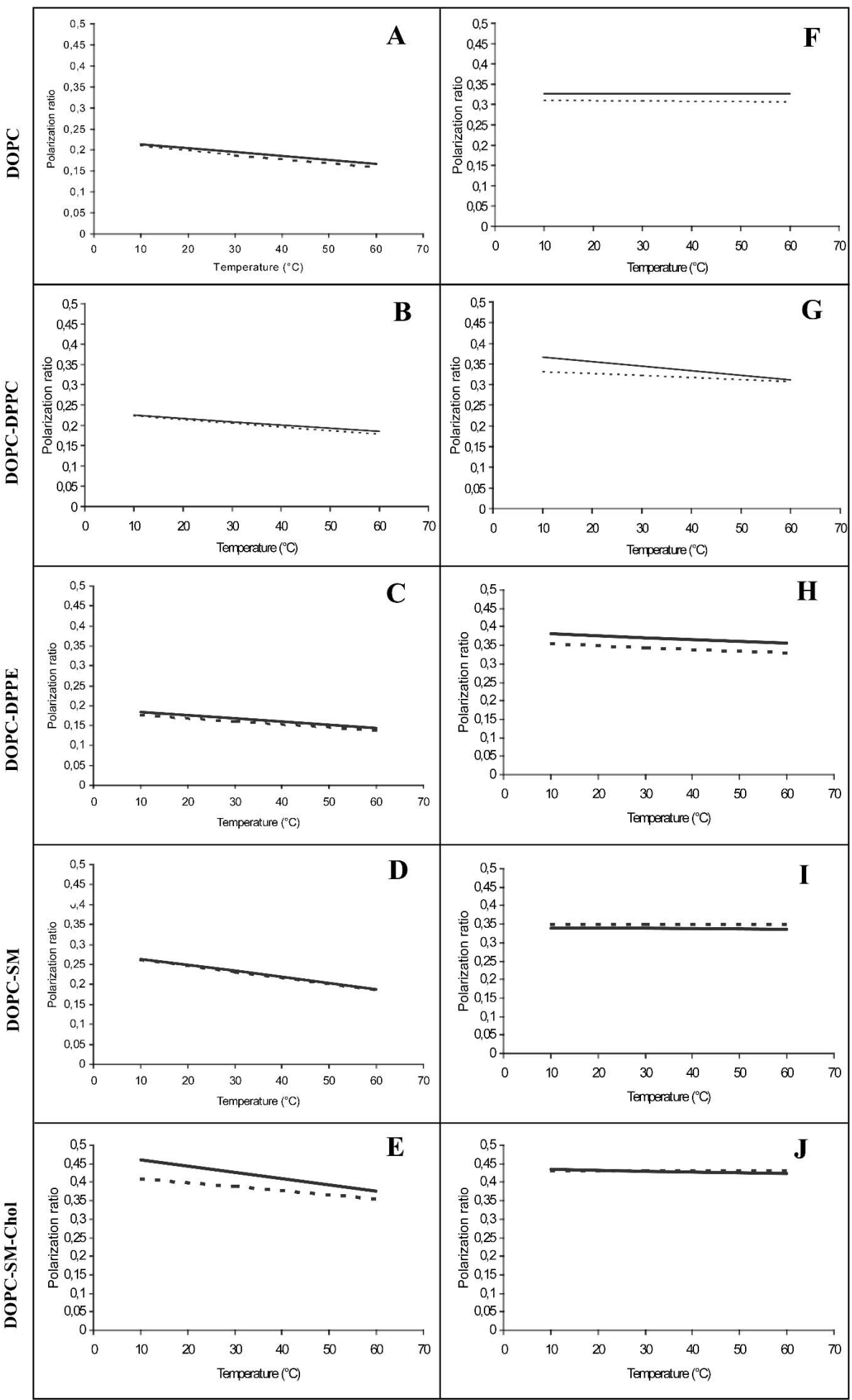

Fig. 5. Effect of azithromycin on DPH (A-E) and TMA-DPH (F-J) fluorescence polarization on lipid vesicles. Liposomes $(0.2 \mathrm{mg} / \mathrm{ml})$ were prepared at $\mathrm{pH} 7.4$ and allowed to incorporate DPH (left panels; A-E) or TMA-DPH (right panels; F-J) at a molar ratio to the lipids of 1:250. Labeled liposomes (full line) were then mixed with azithromycin $(132 \mu \mathrm{M}$; dashed lines) and incubated at $37^{\circ} \mathrm{C}$ for $30 \mathrm{~min}$. Liposomes (DOPC [1:1 mol:mol] [A,F]; DPPC:DOPC [1:1 mol:mol] [B,G]; DPPE:DOPC [1:1 mol:mol] [C,H]; SM:DOPC [1:1 mol:mol] [D,I]; SM:Chol:DOPC [1:1:1 mol:mol:mol] [E,J]) were then brought to $60^{\circ} \mathrm{C}$ in $15 \mathrm{~min}$ and stabilized at this temperature during $5 \mathrm{~min}$ before starting the measurements, during which the samples were cooled down to $10^{\circ} \mathrm{C}$ at a rate of $50^{\circ} \mathrm{C} / \mathrm{h}$. Data shown are representative of experiments that were reproduced four times for DPH and twice for TMA-DPH. 


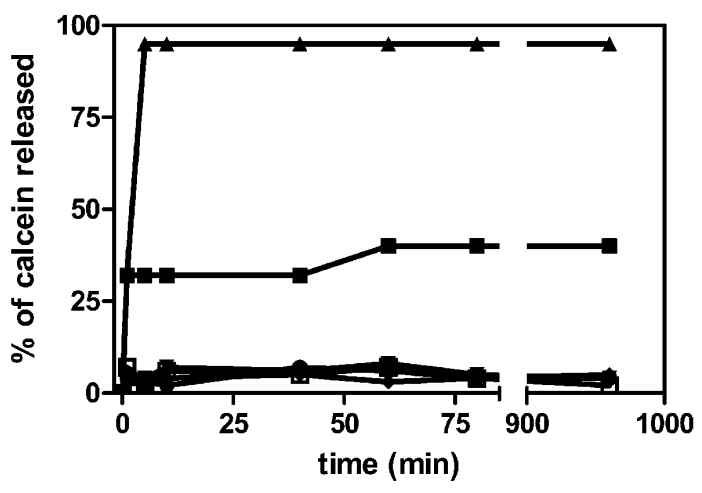

Fig. 6. Effect of azithromycin on the calcein release from liposomes. Liposomes $(50 \mu \mathrm{M})$ (DOPC $\mathbf{\square}$; DPPC:DOPC [1:1 mol:mol] $\mathbf{\nabla}$; DPPE:DOPC [1:1 mol:mol] $\bullet$ SM:DOPC [1:1 mol:mol] $\bullet$; SM:Chol:DOPC [1:1:1 mol:mol:mol] $\square$ ) were incubated with azithromycin $(7 \mu \mathrm{M})$ or mellitin $\boldsymbol{\Delta}$ (positive control whatever the lipidic composition of the vesicles). Each point is the mean value of three independent experiments, but the SD $(<2.5 \%)$ are not shown for sake of clarity.

the boundary regions, the interdigitated membrane being characterized by a thinner structure and more rigid hydrocarbon regions than its noninterdigitated counterpart (75). The effect induced by azithromycin, however, is probably not sufficient to lead to instabilities and structure defects in the boundary regions that would be expected to cause increased permeability. The lack of effect of azithromycin on the fluidity of hydrophobic region in DPPC:DOPC, DPPE:DOPC, SM:DOPC models and the higher rigidity of SM:Chol:DOPC model, even in presence of azithromycin, could be related to the inability of azithromycin to alter membrane permeability. This absence of effect could also be related to the lack of fusogenic effect of azithromycin on negatively charged liposomes (70).

In conclusion, using AFM, fluorescence polarization studies, and equilibrium dialysis, we showed that the interaction of a macrolide antibiotic, azithromycin, with lipids (DPPC:DOPC or DPPE:DOPC or SM:DOPC or SM:Chol: DOPC) is highly dependent on the nature of the lipids. The main finding of this work is that azithromycin perturbs the organization of DPPC and DPPE gel domains in DOPC fluidphase but has no effect on SM or SM:cholesterol domains. We suggested that these observations are related to i) the increase of the membrane fluidity at the hydrophilic/hydrophobic interface of DPPC:DOPC and DPPE:DOPC models without effect in vesicles containing sphingomyelin and/or ii) the rigidifying effect and the competition for space in the domain interfaces induced by cholesterol. The effect of azithromycin on the organization of lipid domains is, however, not sufficient to induce membrane permeabilization.

\section{ACKNOWLEDGMENTS}

Y. F. D. is a research associate of the Belgian National Foundation for Scientific Research (FNRS). This work was supported by the Belgian Fonds de la Recherche Scientifique Médicale (grants no. 3.4589.96 and no. 3.4546.02 to M-P. M-L.), by the Fonds Speciaux de Recherche (FSR 2002) of the Université catholique de Louvain (to M-P. M-L.), and by Région wallonne (grant no. 115020 to M-P. M-L. and Y. D.). The authors thank Prof. E. Gaigneaux for the use of the atomic force microscope.

\section{REFERENCES}

1. S. J. Singer and G. L. Nicolson. The fluid mosaic model of the structure of cell membranes. Science 175:720-731 (1972).

2. P. Somerharju, J. A. Virtanen, and K. H. Cheng. Lateral organisation of membrane lipids. The superlattice view. Biochim. Biophys. Acta 1440:32-48 (1999).

3. S. Pfeffer. Membrane domains in the secretory and endocytic pathways. Cell 112:507-517 (2003).

4. B. van Deurs, K. Roepstorff, A. M. Hommelgaard, and K. Sandvig. Caveolae: anchored, multifunctional platforms in the lipid ocean. Trends Cell Biol. 13:92-100 (2003).

5. J. C. Holthuis, G. van Meer, and K. Huitema. Lipid microdomains, lipid translocation and the organization of intracellular membrane transport (Review). Mol. Membr. Biol. 20:231-241 (2003)

6. A. D. Dergunov, J. Taveirne, B. Vanloo, H. Caster, and M. Rosseneu. Structural organization of lipid phase and protein-lipid interface in apolipoprotein-phospholipid recombinants: influence of cholesterol. Biochim. Biophys. Acta 1346:131-146 (1997).

7. K. Jorgensen, J. H. Ipsen, O. G. Mouritsen, and M. J. Zuckermann. The effect of anaesthetics on the dynamic heterogeneity of lipid membranes. Chem. Phys. Lipids 65:205-216 (1993).

8. K. Jorgensen, J. H. Ipsen, O. G. Mouritsen, D. Bennett, and M. J. Zuckermann. The effects of density fluctuations on the partitioning of foreign molecules into lipid bilayers: application to anaesthetics and insecticides. Biochim. Biophys. Acta 1067:241253 (1991).

9. T. Soderlund, J. Y. Lehtonen, and P. K. Kinnunen. Interactions of cyclosporin A with phospholipid membranes: effect of cholesterol. Mol. Pharmacol. 55:32-38 (1999).

10. J. J. Wenz and F. J. Barrantes. Steroid structural requirements for stabilizing or disrupting lipid domains. Biochemistry 42:1426714276 (2003).

11. A. B. Hendrich, O. Wesolowska, and K. Michalak. Trifluoperazine induces domain formation in zwitterionic phosphatidylcholine but not in charged phosphatidylglycerol bilayers. Biochim. Biophys. Acta 1510:414-425 (2001).

12. A. Schanck, M. P. Mingeot-Leclercq, P. M. Tulkens, D. Carrier, I. C. Smith, and H. C. Jarrell. Interactions of aminoglycoside antibiotics with phospholipids. A deuterium nuclear magnetic resonance study. Chem. Phys. Lipids 62:153-163 (1992).

13. D. Tyteca, A. Schanck, Y. F. Dufrene, M. Deleu, P. J. Courtoy, P. M. Tulkens, and M. P. Mingeot-Leclercq. The macrolide antibiotic azithromycin interacts with lipids and affects membrane organization and fluidity: studies on Langmuir-Blodgett monolayers, liposomes and J774 macrophages. J. Membr. Biol. 192: 203-215 (2003).

14. D. Tyteca, S. P. Van Der, M. Mettlen, F. Van Bambeke, P. M. Tulkens, M. P. Mingeot-Leclercq, and P. J. Courtoy. Azithromycin, a lysosomotropic antibiotic, has distinct effects on fluid-phase and receptor-mediated endocytosis, but does not impair phagocytosis in J774 macrophages. Exp. Cell Res. 281:86-100 (2002).

15. D. Tyteca, S. P. Van Der, F. Van Bambeke, K. Leys, P. M. Tulkens, P. J. Courtoy, and M. P. Mingeot-Leclercq. Azithromycin, a lysosomotropic antibiotic, impairs fluid-phase pinocytosis in cultured fibroblasts. Eur. J. Cell Biol. 80:466-478 (2001).

16. J. C. Hooton, C. S. German, S. Allen, M. C. Davies, C. J. Roberts, S. J. Tendler, and P. M. Williams. An atomic force microscopy study of the effect of nanoscale contact geometry and surface chemistry on the adhesion of pharmaceutical particles. Pharm. Res. 21:953-961 (2004).

17. K. Six, J. Murphy, I. Weuts, D. Q. Craig, G. Verreck, J. Peeters, M. Brewster, and M. G. Van den. Identification of phase separation in solid dispersions of itraconazole and Eudragit E100 using microthermal analysis. Pharm. Res. 20:135-138 (2003).

18. Y. F. Dufrene and G. U. Lee. Advances in the characterization of supported lipid films with the atomic force microscope. Biochim. Biophys. Acta 1509:14-41 (2000).

19. A. Engel and D. J. Muller. Observing single biomolecules at work with the atomic force microscope. Nat. Struct. Biol. 7:715-718 (2000). 
20. M. P. Mingeot-Leclercq, J. Piret, R. Brasseur, and P. M. Tulkens. Effect of acidic phospholipids on the activity of lysosomal phospholipases and on their inhibition by aminoglycoside antibioticsI. Biochemical analysis. Biochem. Pharmacol. 40:489-497 (1990).

21. R. D. Kaiser and E. London. Location of diphenylhexatriene (DPH) and its derivatives within membranes: comparison of different fluorescence quenching analyses of membrane depth. Biochemistry 37:8180-8190 (1998).

22. B. R. Lentz. Use of fluorescent probes to monitor molecular order and motions within liposome bilayers. Chem. Phys. Lipids 64:99-116 (1993).

23. S. Kitagawa, M. Matsubayashi, K. Kotani, K. Usui, and F. Kametani. Asymmetry of membrane fluidity in the lipid bilayer of blood platelets: fluorescence study with diphenylhexatriene and analogs. J. Membr. Biol. 119:221-227 (1991).

24. J. N. Weinstein, S. Yoshikami, P. Henkart, R. Blumenthal, and W. A. Hagins. Liposome-cell interaction: transfer and intracellular release of a trapped fluorescent marker. Science 195:489-492 (1977).

25. F. Van Bambeke, M. P. Mingeot-Leclercq, A. Schanck, R. Brasseur, and P. M. Tulkens. Alterations in membrane permeability induced by aminoglycoside antibiotics: studies on liposomes and cultured cells. Eur. J. Pharmacol. 247:155-168 (1993).

26. G. R. Bartlett. Phosphorus assay in column chromatography. $J$. Biol. Chem. 234:466-468 (1959).

27. S. W. Hui, R. Viswanathan, J. A. Zasadzinski, and J. N. Israelachvili. The structure and stability of phospholipid bilayers by atomic force microscopy. Biophys. J. 68:171-178 (1995).

28. N. C. Santos, E. Ter Ovanesyan, J. A. Zasadzinski, and M. A. Castanho. Reconstitution of phospholipid bilayer by an atomic force microscope tip. Biophys. J. 75:2119-2120 (1998).

29. O. G. Mouritsen and K. Jorgensen. Dynamical order and disorder in lipid bilayers. Chem. Phys. Lipids 73:3-25 (1994).

30. M. M. Baksh, M. Jaros, and J. T. Groves. Detection of molecular interactions at membrane surfaces through colloid phase transitions. Nature 427:139-141 (2004).

31. D. A. Middleton, D. G. Reid, and A. Watts. Combined quantitative and mechanistic study of drug-membrane interactions using a novel 2H NMR approach. J. Pharm. Sci. 93:507-514 (2004).

32. C. Matos, J. L. Lima, S. Reis, A. Lopes, and M. Bastos. Interaction of antiinflammatory drugs with EPC liposomes: calorimetric study in a broad concentration range. Biophys. J. 86:946-954 (2004).

33. B. Lopez-Garcia, J. F. Marcos, C. Abad, and E. Perez-Paya. Stabilisation of mixed peptide/lipid complexes in selective antifungal hexapeptides. Biochim. Biophys. Acta 1660:131-137 (2004).

34. H. Lygre, G. Moe, and H. Holmsen. Interaction of ibuprofen with eukaryotic membrane lipids. Acta Odontol. Scand. 61:303-309 (2003).

35. P. Suomalainen, C. Johans, T. Soderlund, and P. K. Kinnunen. Surface activity profiling of drugs applied to the prediction of blood-brain barrier permeability. J. Med. Chem. 47:1783-1788 (2004).

36. A. A. Hidalgo, W. Caetano, M. Tabak, and O. N. Oliveira Jr. Interaction of two phenothiazine derivatives with phospholipid monolayers. Biophys. Chem. 109:85-104 (2004).

37. T. Harder, P. Scheiffele, P. Verkade, and K. Simons. Lipid domain structure of the plasma membrane revealed by patching of membrane components. J. Cell Biol. 141:929-942 (1998).

38. H. W. Huang, E. M. Goldberg, and R. Zidovetzki. Ceramide induces structural defects into phosphatidylcholine bilayers and activates phospholipase A2. Biochem. Biophys. Res. Commun. 220:834-838 (1996).

39. T. Kobayashi, M. H. Beuchat, J. Chevallier, A. Makino, N. Mayran, J. M. Escola, C. Lebrand, P. Cosson, T. Kobayashi, and J. Gruenberg. Separation and characterization of late endosomal membrane domains. J. Biol. Chem. 277:32157-32164 (2002).

40. E. C. Miller and G. M. Helmkamp Jr. Exposure of phosphatidylinositol transfer proteins to sphingomyelin-cholesterol membranes suggests transient but productive interactions with raftlike, liquid-ordered domains. Biochemistry 42:13250-13259 (2003).

41. V. Schram and T. E. Thompson. Influence of the intrinsic membrane protein bacteriorhodopsin on gel-phase domain topology in two-component phase-separated bilayers. Biophys. J. 72:22172225 (1997)

42. K. H. Kim, T. Ahn, and C. H. Yun. Membrane properties induced by anionic phospholipids and phosphatidylethanolamine are critical for the membrane binding and catalytic activity of human cytochrome P450 3A4. Biochemistry 42:15377-15387 (2003).

43. M. L. Fanani, S. Hartel, R. G. Oliveira, and B. Maggio. Bidirectional control of sphingomyelinase activity and surface topography in lipid monolayers. Biophys. J. 83:3416-3424 (2002).

44. L. Yang and M. Glaser. Formation of membrane domains during the activation of protein kinase C. Biochemistry 35:13966-13974 (1996).

45. K. M. Maloney, M. Grandbois, C. Salesse, D. W. Grainger, and A. Reichert. Membrane microstructural templates for enzyme domain formation. J. Mol. Recognit. 9:368-374 (1996).

46. E. S. Stuart, W. C. Webley, and L. C. Norkin. Lipid rafts, caveolae, caveolin-1, and entry by Chlamydiae into host cells. Exp. Cell Res. 287:67-78 (2003).

47. W. B. Huttner and J. Zimmerberg. Implications of lipid microdomains for membrane curvature, budding and fission. Curr. Opin. Cell Biol. 13:478-484 (2001).

48. M. A. del Pozo, N. B. Alderson, W. B. Kiosses, H. H. Chiang, R. G. Anderson, and M. A. Schwartz. Integrins regulate Rac targeting by internalization of membrane domains. Science 303:839_ 842 (2004).

49. C. Bezombes, G. Laurent, and J. P. Jaffrezou. Implication of raft microdomains in drug induced apoptosis. Curr. Med. Chem. AntiCanc. Agents 3:263-270 (2003).

50. G. M. Humphries and J. P. Lovejoy. Lateral phase separation of phospholipids as a basis for increased permeability of membranes towards fluorescein and other chemical species. J. Membr. Biol. 80:249-256 (1984).

51. M. A. Singer and M. K. Jain. Interaction of four local anesthetics with phospholipid bilayer membranes: permeability effects and possible mechanisms. Can. J. Biochem. 58:815-821 (1980).

52. O. G. Mouritsen and K. Jorgensen. A new look at lipid-membrane structure in relation to drug research. Pharm. Res. 15:1507-1519 (1998).

53. M. C. Giocondi, P. E. Milhiet, P. Dosset, and C. Le Grimellec. Use of cyclodextrin for AFM monitoring of model raft formation. Biophys. J. 86:861-869 (2004).

54. D. P. Siegel. Inverted micellar intermediates and the transitions between lamellar, cubic, and inverted hexagonal lipid phases. II. Implications for membrane-membrane interactions and membrane fusion. Biophys. J. 49:1171-1183 (1986).

55. M. P. Andrich and J. M. Vanderkooi. Temperature dependence of 1,6-diphenyl-1,3,5-hexatriene fluorescence in phophoslipid artificial membranes. Biochemistry 15:1257-1261 (1976).

56. B. R. Lentz, Y. Barenholz, and T. E. Thompson. Fluorescence depolarization studies of phase transitions and fluidity in phospholipid bilayers. 1. Single component phosphatidylcholine liposomes. Biochemistry 15:4521-4528 (1976).

57. B. R. Lentz, Y. Barenholz, and T. E. Thompson. Fluorescence depolarization studies of phase transitions and fluidity in phospholipid bilayers. 2 Two-component phosphatidylcholine liposomes. Biochemistry 15:4529-4537 (1976).

58. F. R. Maxfield. Plasma membrane microdomains. Curr. Opin. Cell Biol. 14:483-487 (2002)

59. C. Dietrich, L. A. Bagatolli, Z. N. Volovyk, N. L. Thompson, M. Levi, K. Jacobson, and E. Gratton. Lipid rafts reconstituted in model membranes. Biophys. J. 80:1417-1428 (2001).

60. B. J. Nichols. GM1-containing lipid rafts are depleted within clathrin-coated pits. Curr. Biol. 13:686-690 (2003).

61. P. Draber and L. Draberova. Lipid rafts in mast cell signaling. Mol. Immunol. 38:1247-1252 (2002).

62. Y. Barenholz. Cholesterol and other membrane active sterols: from membrane evolution to "rafts". Prog. Lipid Res. 41:1-5 (2002).

63. X. M. Li, M. M. Momsen, H. L. Brockman, and R. E. Brown Sterol structure and sphingomyelin acyl chain length modulate lateral packing elasticity and detergent solubility in model membranes. Biophys. J. 85:3788-3801 (2003).

64. H. A. Rinia, M. M. Snel, J. P. van der Eerden, and B. de Kruijff 
Visualizing detergent resistant domains in model membranes with atomic force microscopy. FEBS Lett. 501:92-96 (2001).

65. P. R. Maulik and G. G. Shipley. Interactions of N-stearoyl sphingomyelin with cholesterol and dipalmitoylphosphatidylcholine in bilayer membranes. Biophys. J. 70:2256-2265 (1996).

66. T. J. McIntosh, S. A. Simon, D. Needham, and C. H. Huang. Structure and cohesive properties of sphingomyelin/cholesterol bilayers. Biochemistry 31:2012-2020 (1992).

67. F. A. Nezil and M. Bloom. Combined influence of cholesterol and synthetic amphiphillic peptides upon bilayer thickness in model membranes. Biophys. J. 61:1176-1183 (1992).

68. D. Needham and R. S. Nunn. Elastic deformation and failure of lipid bilayer membranes containing cholesterol. Biophys. J. 58: 997-1009 (1990).

69. E. Evans and D. Needham. Giant vesicle bilayers composed of mixtures of lipids, cholesterol and polypeptides. Thermomechanical and (mutual) adherence properties. Faraday Discuss. Chem. Soc. 267-280 (1986).

70. J. P. Montenez, F. Van Bambeke, J. Piret, A. Schanck, R. Brasseur, P. M. Tulkens, and M. P. Mingeot-Leclercq. Interaction of the macrolide azithromycin with phospholipids. II. Biophysical and computer-aided conformational studies. Eur. J. Pharmacol. 314:215-227 (1996)

71. J. P. Montenez, F. Van Bambeke, J. Piret, R. Brasseur, P. M. Tulkens, and M. P. Mingeot-Leclercq. Interactions of macrolide antibiotics (Erythromycin A, roxithromycin, erythromycylamine [Dirithromycin], and azithromycin) with phospholipids: computer-aided conformational analysis and studies on acellular and cell culture models. Toxicol. Appl. Pharmacol. 156:129-140 (1999).

72. F. Van Bambeke, J. P. Montenez, J. Piret, P. M. Tulkens, P. J. Courtoy, and M. P. Mingeot-Leclercq. Interaction of the macrolide azithromycin with phospholipids. I. Inhibition of lysosomal phospholipase A1 activity. Eur. J. Pharmacol. 314:203-214 (1996).

73. R. Brasseur, G. Laurent, J. M. Ruysschaert, and P. Tulkens. Interactions of aminoglycoside antibiotics with negatively charged lipid layers. Biochemical and conformational studies. Biochem. Pharmacol. 33:629-637 (1984).

74. M. P. Mingeot-Leclercq, A. Schanck, M. F. Ronveaux-Dupal, M. Deleers, R. Brasseur, J. M. Ruysschaert, G. Laurent, and P. M. Tulkens. Ultrastructural, physico-chemical and conformational study of the interactions of gentamicin and bis(betadiethylaminoethylether) hexestrol with negatively-charged phospholipid layers. Biochem. Pharmacol. 38:729-741 (1989).

75. J. L. Slater, C. H. Huang, and I. W. Levin. Interdigitated bilayer packing motifs: Raman spectroscopic studies of the eutectic phase behavior of the 1-stearoyl-2-caprylphosphatidylcholine/ dimyristoylphosphatidylcholine binary mixture. Biochim. Biophys. Acta 1106:242-250 (1992). 